\title{
A Treatment of the Absorption Spectrum for a Multiphoton $V$-Type Three-Level Atom Interacting with a Squeezed Coherent Field in the Presence of Nonlinearities
}

\author{
F. K. Faramawy \\ Mathematics Department, Faculty of Science, Al-Azhar University, Nasr City, Cairo 11884, Egypt \\ Correspondence should be addressed to F. K. Faramawy, fkf54@yahoo.com
}

Received 15 May 2011; Revised 9 October 2011; Accepted 27 October 2011

Academic Editor: Ch. Tsitouras

Copyright (C) 2011 F. K. Faramawy. This is an open access article distributed under the Creative Commons Attribution License, which permits unrestricted use, distribution, and reproduction in any medium, provided the original work is properly cited.

\begin{abstract}
We study the interaction of a three-level atom with a single mode field through multiphoton transition in a cavity, taking explicitly into account the existence of forms of nonlinearities of both the field and the intensity-dependent atom-field coupling. The analytical forms of the absorption spectrum is calculated using the dressed states of the system. The effects of photon multiplicities, mean photon number, detuning, Kerr-like medium, and the intensity-dependent coupling functional on the absorption spectrum are analyzed.
\end{abstract}

\section{Introduction}

The spectrum of spontaneous emission of a $V$-configuration three-level atom, whose two upper levels are coupled by a classical field and their energy spacing is much larger than the spontaneous emission widths has been investigated [1]. It has been shown that the spontaneously generated interference can induce the spectrum to exhibit six peaks and depend on the phase of the classical field. The effects of a broadband squeezed vacuum on three-level atoms at different configurations $(\Lambda, V$, and $\Xi$ configurations) have also been investigated [2-5]. Further work has also been done to study the resonance fluorescence spectra of three-level atoms interacting with two coherent lasers and two independent squeezed vacuum [3-5]. The fluorescence spectrum for a strongly driven three-level system in which one of the two one-photon transition is coupled to a finite-bandwidth squeezed vacuum field has been examined [4]. Quantum interference effects in resonance fluorescence and absorption spectra of a $V$-type three-level atom damped by a broadband squeezed vacuum studied in [6]. 
In recent years, there has been tremendous progress in the ability to generate states of the electromagnetic field with manifestly quantum or nonclassical characteristics experimentally [7-9]. Because the squeezed coherent states are experimentally available, we use it in this paper. Squeezed states of light are nonclassical states for which the fluctuations in one of two quadrature phase amplitudes of the electromagnetic field drop below the level of fluctuations associated with the vacuum state of the field. Squeezed states therefore provide a field which is in some sense quieter than the vacuum state and hence can be employed to improve measurement precision beyond the standard quantum limits [7].

The goal of this paper is to shed some light on the absorption spectrum for a general three-level system. The model we will consider is consisting of a single $V$-type three-level atom interacting with a multiphoton one mode field in a perfect cavity, including acceptable kinds of nonlinearities of both the field and the intensity-dependent atom-field coupling. To reach our goal it is more convenient to use exact expression for the unitary operator $U(t)$ in the frame of the dressed state formalism. This will be considered in Section 2. In Section 3 we employ the analytical results obtained, by using the finite double-Fourier transform of the two-time field correlation function, to find an analytical expression for the absorption spectrum. By a numerical computation, we examine the influence of photon multiplicities, mean photon number, detuning parameters, the functional dependence of the coupling as well as the nonlinearity parameter on the absorption spectrum in Section 4. Finally, the conclusions are summarized in Section 5.

\section{Formulation of the Problem}

The Hamiltonian of the system in the rotating-wave approximation is of the form $(\hbar=1)$

$$
\begin{gathered}
H=H_{0}+H_{\text {in }} \\
H_{0}=\sum_{j=1}^{3} \omega_{j} \sigma_{j, j}+\Omega \widehat{a}^{+} \widehat{a} .
\end{gathered}
$$

The operators $\widehat{a}$ and $\hat{a}^{+}$are the boson operators for the field satisfying $\left[\widehat{a}, \widehat{a}^{+}\right]=1$. Where $\omega_{1}, \omega_{2}$, and $\omega_{3}$ are the atomic energy levels $\left(\omega_{1}>\omega_{2}>\omega_{3}\right)$ and $\Omega$ is the field frequency, with the detuning parameters $\Delta_{1}$ and $\Delta_{2}$ given by

$$
\Delta_{1}=-k \Omega+\left(\omega_{1}-\omega_{3}\right), \quad \Delta_{2}=-k \Omega+\left(\omega_{2}-\omega_{3}\right) .
$$

The interaction part of the Hamiltonian in the presence of an arbitrary nonlinear medium, via multiphoton process $k$ can be written as

$$
\begin{aligned}
H_{\text {in }}= & \Re\left(\hat{a}^{\dagger} \widehat{a}\right)+\lambda_{1}\left(\sigma_{13} f_{1}\left(\hat{a}^{\dagger} \widehat{a}\right) \hat{a}^{k}+\widehat{a}^{+k} f_{1}\left(\hat{a}^{\dagger} \widehat{a}\right) \sigma_{31}\right) \\
& +\lambda_{2}\left(\sigma_{23} f_{2}\left(\hat{a}^{\dagger} \hat{a}\right) \hat{a}^{k}+\widehat{a}^{+k} f_{2}\left(\hat{a}^{\dagger} \widehat{a}\right) \sigma_{32}\right) .
\end{aligned}
$$




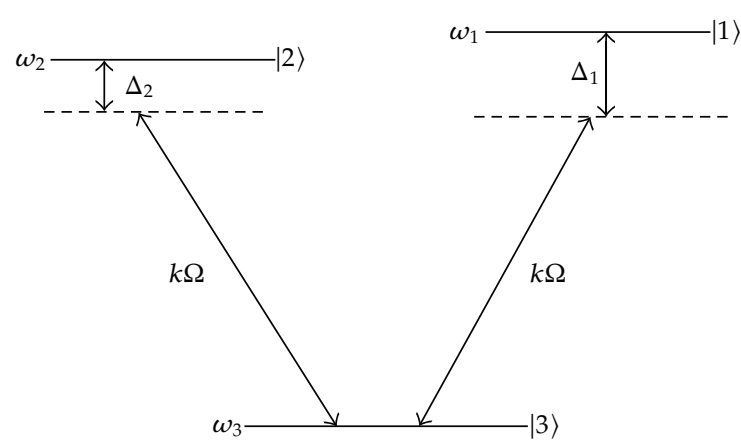

Figure 1: Energy level diagram for a $V$-type three-level atom with $k$-photon detuning $\Delta_{1}, \Delta_{2}$.

$\mathfrak{R}\left(\widehat{a}^{\dagger} \widehat{a}\right)$ and $f\left(\widehat{a}^{\dagger} \widehat{a}\right)$ are Hermitian operators functions of photon number operators, such that $\lambda_{1} f_{1}\left(\widehat{a}^{\dagger} \widehat{a}\right)$ and $\lambda_{2} f_{2}\left(\widehat{a}^{\dagger} \widehat{a}\right)$ represent arbitrary intensity-dependent atom-field coupling, while $\mathfrak{R}\left(\hat{a}^{\dagger} \widehat{a}\right)$ denotes the one-mode field nonlinearity which can model Kerr-like medium nonlinearity as will be discussed later. The operators $\sigma_{i j}$ satisfy the following commutation relations $\left[\sigma_{i j}, \sigma_{\kappa l}\right]=\sigma_{i l} \delta_{j \kappa}-\sigma_{\kappa j} \delta_{i l},\left[\hat{a}, \sigma_{i j}\right]=0$ (Figure 1 ).

The initial state $\left|\Psi(0)_{\mathrm{AF}}\right\rangle$ of the combined atom-field system may be written as

$$
\left|\Psi(0)_{\mathrm{AF}}\right\rangle=\left|\Psi(0)_{\mathrm{A}}\right\rangle \otimes\left|\Psi(0)_{\mathrm{F}}\right\rangle,
$$

where $\left|\Psi(0)_{\mathrm{A}}\right\rangle=|1\rangle\langle 1|$ the initial state of the atom and $\left|\Psi(0)_{\mathrm{F}}\right\rangle=|\Theta\rangle\langle\Theta|$ is the initial state of the field. The initial state $|\Theta\rangle=\Sigma p(n)|n\rangle$ where the probability amplitude $p(n)$ is defined in the usual manner as $p(n)=\langle n \mid \Theta\rangle$.

The time evolution between the atom and the field is defined by the unitary evolution operator generated by $H$. Thus $U(t)$ is given by

$$
U(t) \equiv \exp (-i H t)
$$

This unitary operator $U(t)$ is written as

$$
U(t)=\sum_{s=0}^{k-1} \exp \left(-i E_{03}^{(s)} t\right)\left|\Phi^{(s)}\right\rangle\left\langle\Phi^{(s)}\left|+\sum_{n=k}^{\infty} \sum_{j=1}^{3} \exp \left(-i E_{j}^{(n)} t\right)\right| \Psi_{j}^{(n)}\right\rangle\left\langle\Psi_{j}^{(n)}\right|
$$

where $j=1,2,3$, and the eigenvalues

$$
\begin{gathered}
E_{03}^{(s)}=\omega_{3}+\Omega s+\Re(s), \quad(s=0,1, \ldots, k-1), \\
E_{j}^{(n)}=-\frac{X_{1}}{3}+\frac{2}{3}\left(\sqrt{X_{1}^{2}-3 X_{2}}\right) \cos \left(\theta_{j}\right), \\
\theta_{j}=\left(\frac{1}{3} \cos ^{-1}\left[\frac{9 X_{1} X_{2}-2 X_{1}^{3}-27 X_{3}}{2\left(X_{1}^{2}-3 X_{2}\right)^{3 / 2}}\right]+(j-1) \frac{2 \pi}{3}\right),
\end{gathered}
$$


with

$$
\begin{aligned}
& X_{1}=-\left(r_{1}+r_{2}+r_{3}\right), \\
& X_{2}=-\left[V_{1}^{2}+V_{2}^{2}-r_{1} r_{2}-r_{1} r_{3}-r_{2} r_{3}\right], \\
& X_{3}=r_{2} V_{1}^{2}+r_{1} V_{2}^{2}-r_{1} r_{2} r_{3}, \\
& r_{1}=\omega_{1}+\Omega n+\Re(n), \\
& r_{2}=\omega_{2}+\Omega n+\Re(n), \\
& r_{3}=\omega_{3}+\Omega(n+k)+\Re(n+k), \\
& V_{1}=\lambda_{1} f_{1}(n) \sqrt{\frac{(n+k) !}{n !},} \quad V_{2}=\lambda_{2} f_{2}(n) \sqrt{\frac{(n+k) !}{n !},}
\end{aligned}
$$

and $\left|\Phi^{(s)}\right\rangle,\left|\Psi_{j}^{(n)}\right\rangle$ are the dressed states of the system associated with the eigenvalues $E_{03}^{(s)}$ and $E_{j}^{(n)},(j=1,2,3)$

$$
\begin{aligned}
& \left|\Phi^{(s)}\right\rangle=|s, 3\rangle, \quad(s=0,1, \ldots, k-1), \\
& \left|\Psi_{j}^{(n)}\right\rangle=\alpha_{j}^{(n)}|n, 1\rangle+\beta_{j}^{(n)}|n, 2\rangle+\gamma_{j}^{(n)}|n+k, 3\rangle,
\end{aligned}
$$

where

$$
\begin{gathered}
\left(\begin{array}{c}
\alpha_{j}^{(n)} \\
\beta_{j}^{(n)} \\
r_{j}^{(n)}
\end{array}\right)=\frac{1}{M}\left(\begin{array}{c}
-V_{1}\left(r_{2}-E_{j}^{(n)}\right) \\
-V_{2}\left(r_{1}-E_{j}^{(n)}\right) \\
\left(r_{1}-E_{j}^{(n)}\right)\left(r_{2}-E_{j}^{(n)}\right)
\end{array}\right) \\
M=\sqrt{\left(r_{1}-E_{j}^{(n)}\right)^{2}\left(r_{2}-E_{j}^{(n)}\right)^{2}+V_{1}^{2}\left(r_{2}-E_{j}^{(n)}\right)^{2}+V_{2}^{2}\left(r_{1}-E_{j}^{(n)}\right)^{2}} .
\end{gathered}
$$

Having obtained the explicit form of the unitary operator $U(t)$, the eigenvalues and the eigenfunctions for the system under consideration, we are therefore in a position to discuss some properties related to the atom or the field, especially the absorption spectrum. 


\section{Absorption Spectrum}

In this section we calculate the absorption spectrum as [5],

$$
\begin{aligned}
A(v)= & \Gamma \int_{0}^{T} d t_{1} \int_{0}^{T} d t_{2} \exp \left[-(\Gamma-i v)\left(T-t_{1}\right)-(\Gamma+i v)\left(T-t_{2}\right)\right] \\
& \times\left\langle\left[\left(\sigma_{13}\left(t_{1}\right)+\sigma_{23}\left(\mathrm{t}_{1}\right)\right),\left(\sigma_{31}\left(t_{2}\right)+\sigma_{32}\left(t_{2}\right)\right)\right]\right\rangle,
\end{aligned}
$$

where $T$ is the interaction time and $\Gamma$ is the bandwidth of the filter. The Fourier transform, of the two-time commutator averaged dipole-dipole correlation, is directly related to the absorption spectrum, where

$$
\begin{aligned}
\left\langle\left[\left(\sigma_{13}\left(t_{1}\right)+\sigma_{23}\left(t_{1}\right)\right),\left(\sigma_{31}\left(t_{2}\right)+\sigma_{32}\left(t_{2}\right)\right)\right]\right\rangle= & \left\langle\left(\sigma_{13}\left(t_{1}\right)+\sigma_{23}\left(t_{1}\right)\right)\left(\sigma_{31}\left(t_{2}\right)+\sigma_{32}\left(t_{2}\right)\right)\right\rangle \\
& -\left\langle\left(\sigma_{31}\left(t_{2}\right)+\sigma_{32}\left(t_{2}\right)\right)\left(\sigma_{13}\left(t_{1}\right)+\sigma_{23}\left(t_{1}\right)\right)\right\rangle .
\end{aligned}
$$

The first term $\left\langle\left(\sigma_{13}\left(t_{1}\right)+\sigma_{23}\left(t_{1}\right)\right)\left(\sigma_{31}\left(t_{2}\right)+\sigma_{32}\left(t_{2}\right)\right)\right\rangle$ is associated with emission processes while the second term $\left\langle\left(\sigma_{31}\left(t_{2}\right)+\sigma_{32}\left(t_{2}\right)\right)\left(\sigma_{13}\left(t_{1}\right)+\sigma_{23}\left(t_{1}\right)\right)\right\rangle$, which has an opposite sign, corresponds to stimulated absorption. In order to calculate the absorption spectrum we need to calculate the two-time commutator of (3.1). The probe absorption coefficient is given by the difference between a stimulated absorption and the emission component. However, in what follows we analyze the case when the atom is initially prepared in it is most excited state, and considering the field is being initially a squeezed coherent state. After carrying out the various operations we obtain the absorption spectrum in the form:

$$
\begin{aligned}
A(v)= & \Gamma \sum_{s=0}^{k-1} \sum_{j=1}^{3}\left|p^{(s)}\right|^{2}\left|\alpha_{j}^{(s)}\right|^{2} \Upsilon\left(E_{03}^{(s)}, E_{j}^{(s)}\right) \\
& \times\left[\left|\alpha_{j}^{(s)}\right|^{2}+\alpha_{j}^{*(s)} \beta_{j}^{(s)}+\alpha_{j}^{(s)} \beta_{j}^{*(s)}+\left|\beta_{j}^{(s)}\right|^{2}\right] \\
& +\Gamma \sum_{n=k}^{\infty} \sum_{i=1}^{3} \sum_{j=1}^{3}\left|p^{(n)}\right|^{2}\left|\alpha_{j}^{(n)}\right|^{2}\left|\gamma_{i}^{(n-k)}\right|^{2} \Upsilon\left(E_{i}^{(n-k)}, E_{j}^{(n)}\right) \\
& \times\left[\left|\alpha_{j}^{(n)}\right|^{2}+\alpha_{j}^{*(n)} \beta_{j}^{(n)}+\alpha_{j}^{(n)} \beta_{j}^{*(n)}+\left|\beta_{j}^{(n)}\right|^{2}\right] \\
& -\Gamma \sum_{n=0}^{\infty} \sum_{i=1}^{3} \sum_{j=1}^{3}\left|p^{(n)}\right|^{2}\left|\alpha_{j}^{(n)}\right|^{2}\left|\gamma_{j}^{(n)}\right|^{2} \Upsilon\left(E_{j}^{(n)}, E_{i}^{(n+1)}\right) \\
& \times\left[\left|\alpha_{i}^{(n+1)}\right|^{2}+\alpha_{i}^{*(n+1)} \beta_{i}^{(n+1)}+\alpha_{i}^{(n+1)} \beta_{i}^{*(n+1)}+\left|\beta_{i}^{(n+1)}\right|^{2}\right],
\end{aligned}
$$

where

$$
\Upsilon(x, y)=\left[\frac{1+\exp (-2 \Gamma T)-2 \exp (-\Gamma T) \cos (v+x-y) T}{\Gamma^{2}+(v+x-y)^{2}}\right] .
$$


Thus the time-averaged absorption spectrum consists of resonant structures which arise from transitions among different dressed states. The final structure of the time-averaged absorption spectrum will depend on the form of the input photon distribution $p^{(n)}$. Due to the quantum interference between component states the oscillations in the cavity field become composed of different component states.

\section{Numerical Results and Discussion}

On the basis of the analytical solution presented in a previous section, we will study numerically the absorption spectrum in a a squeezed coherent initial field. The photon number distribution for a squeezed coherent state $[7,10]$ can be written as

$$
\left|P_{n}\right|^{2}=s \frac{(\tanh r)^{n}}{2^{n} n ! \cosh r}\left|H_{n}\left(\frac{\varepsilon}{\sqrt{2 \cosh r \sinh r}}\right)\right|^{2} \exp \left[-|\varepsilon|^{2}+\tanh r \operatorname{Re}(\varepsilon)^{2}\right]
$$

where $\varepsilon=\alpha \cosh r+\alpha^{*} \sinh r, \alpha=|\alpha| \exp (i \varsigma)$, and $H_{n}$ is the Hermite polynomial. We suppose here the minor axis of the ellipse, representing the direction of squeezing, parallel to the coordinate of the field oscillator. The initial phase $\varsigma$ of $\alpha$ is the angle between the direction of coherent excitation and the direction of squeezing. The mean photon number of this field is equal to $\bar{n}=|\alpha|^{2}+\sinh ^{2} r$. Putting $r=0$ we get the photon distribution for an initial coherent state with $\bar{n}=|\alpha|^{2}$, whereas for $\alpha=0$ the photon distribution for an initial squeezed vacuum state with $\bar{n}=\sinh ^{2} r$ is recovered. The latter distribution is oscillatory with zeros for odd $n$.

In general, the spectrum attains negative and positive values: a negative value represents amplification and a positive value represents absorption. Since the expression in (3.4) depends on the difference in population between the upper and the lower energies of the dressed states, one can easily predict that at high intensities the absorption spectrum exhibits an equal number of absorbing and amplifying components. As we mentioned before the total components appearing spectrum are proportional to the difference between the absorption and emission processes occurring during the transit time $T$. Transitions to atomic levels and the dressed states exhibit a structure in absorption spectrum whose components being either absorbing or amplifying.

\subsection{Effect of Multiplicity and Mean Photon Number}

For $k=1$, we observe that the central peak surrounded by two hole-burning and symmetric side peaks giving absorption and hole-burning giving amplification degenerate around the central line for small mean photon number as shown Figure 2(A)-(a). But, the situation is changed for $k>1$, where the central structure disappear, and we observe a number of symmetric side peaks and hole burning, which demonstrate absorption and amplification, respectively, degenerate around the central line, see Figures $2(\mathrm{~A})-(\mathrm{b}, \mathrm{c})$. As the mean photon number and variance increases not only the depth of all side amplification and the height of all side absorption peaks decrease but also all amplification and absorption peaks move away from the central line see Figure 2(B). Furthermore, not only the range of the spectrum and the number of the spectral component increases as the mean photon number increases but also as $k$ increases (compare frames in Figure 2). Also, the depth of all side amplification and 


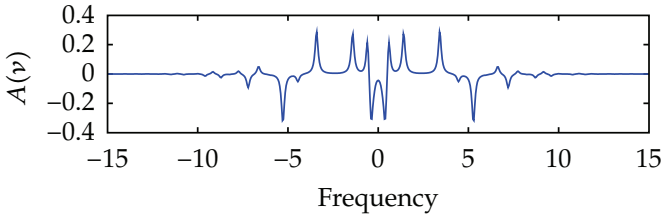

(a)

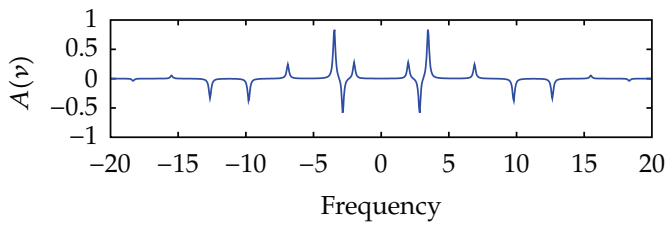

(b)

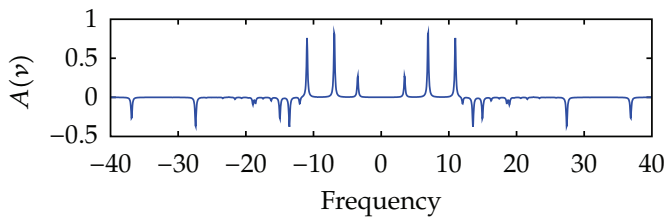

(c)

(A) Squeezed coherent field

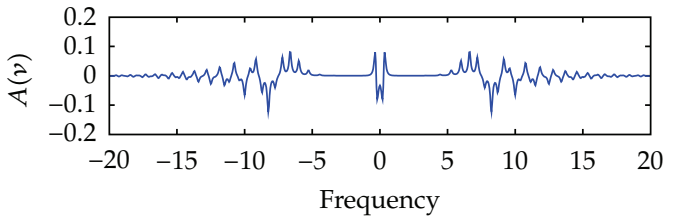

(a)

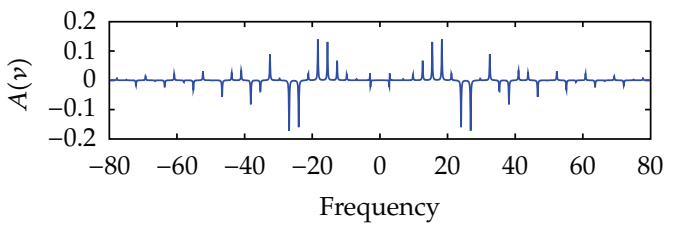

(b)

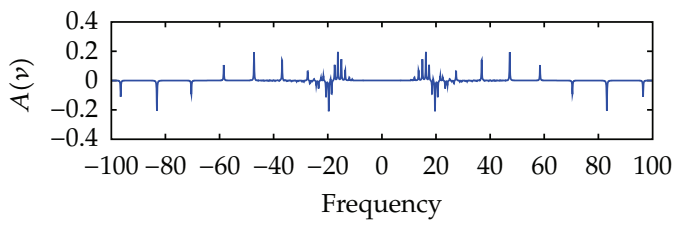

(c)

(B) Squeezed coherent field

Figure 2: The evolution of the function $A(v)$ in a perfect cavity as a function of $(v-k \Omega) / \sqrt{\lambda_{1} \lambda_{2}}$ with $\lambda_{1,2}=1, \Delta_{1,2}=0, x=0, \Gamma=0.1, \varsigma=0, f_{1,2}(n)=1, T=100$ and (a) $k=1$, (b) $k=2$, (c) $k=3$ with (1) $r=1$, $\alpha=1,(2) r=1.7, \alpha=\sqrt{5}$.

the height of all side absorption peaks decrease as soon as the mean photon number increase for all values of $k$.

\subsection{Effect of Detuning}

In Figure 3 we display the absorption spectrum $A(v)$ for different values of the detuning parameters $\Delta_{1}, \Delta_{2}$. We observe that the detuning plays a crucial role in the behavior of the absorption spectrum. For all values of $k$ Figure 3(A) exhibits asymmetric amplification and absorption elements for small values of the detuning parameters $\Delta_{1}$ and $\Delta_{2}$. While interesting modifications are observed, for large detuning parameters, there is strong absorption with minimal amplification, and the peaks are shifted to right side, and we have only strong absorption elements, see Figure 3(B)-(a). This phenomena disappears clearly as $k$ increase, where at $k$ greater than one we still note strong absorption and amplification in right side while minimal absorption and amplification in the left side, Figures $3(B)-(b, c)$. Finally, the heights of the spectral component depend on the values of the detuning parameters and the photon multiplicities $k$.

\subsection{Effect of Kerr Medium}

Now we will turn our attention to the effect on the spectrum $A(v)$ of the nonlinearity of the field with a Kerr-type medium due to the term $\mathfrak{R}(n)$ being taken in the form $\chi_{n}(n-1)$, where $x$ is related to the third-order nonlinear susceptibility. In fact the optical Kerr effect is one 


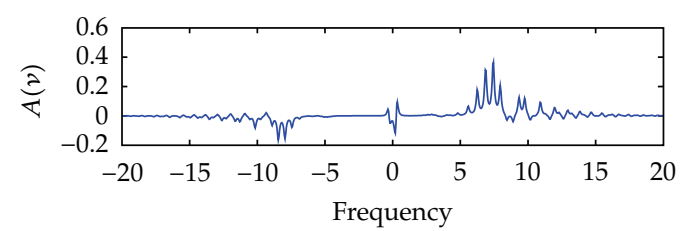

(a)

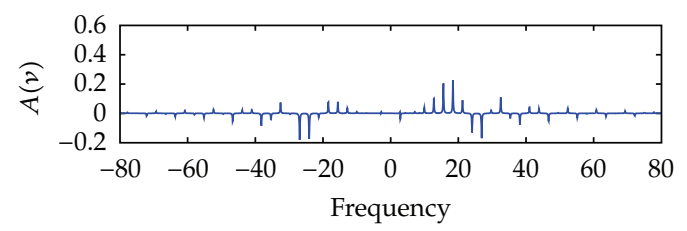

(b)

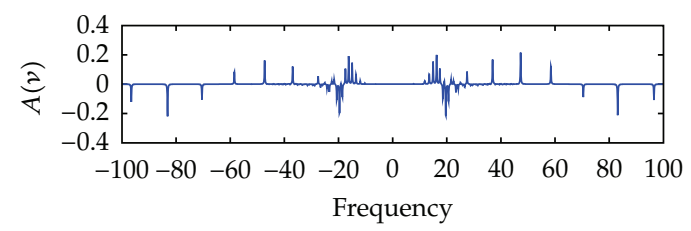

(c)

(A) Squeezed coherent field

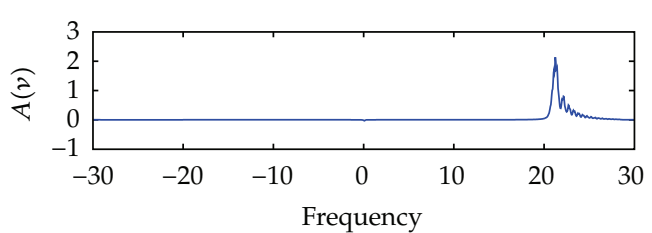

(a)

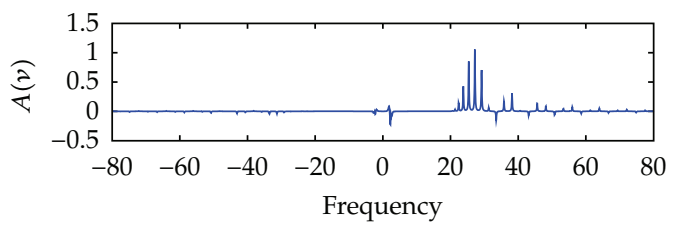

(b)

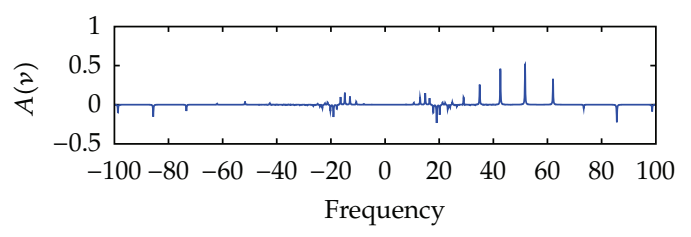

(c)

(B) Squeezed coherent field

Figure 3: The same as Figure 2(B) but with (1) $\Delta_{1}=5, \Delta_{2}=4$, (2) $\Delta_{1}=\Delta_{2}=20$.

of the most extensively studied phenomenon in the field of nonlinear optics because of its applications. The addition of the Kerr-like medium parameter to the problem adds asymmetry to the spectrum as can be seen from comparison of the cases considered in Figure 4 with Figure 2(B). Also, it is to be remarked that the amplitude of the side-bands decrease. As $x$ increases the amplification and absorption element in the left side increase and emerge while on the right side hand elements decrease, and the central line is pushed away to the left side. For large values of $X$ there are no amplification and the spectrum tends to a single absorption element in the left hand side see Figure 4(B). This phenomenon disappears clearly as $k$ increases. While for large $k$ (i.e., $k=3$ ) we still observe a number of strong amplification and absorption peaks in left side, while minimal amplification and absorption peaks redistributed in right side, see Figure 4(B)-(c). Finally, it is interesting to note that Kerr medium has an effect opposite to the effect of the detuning (compare Figure 4 to Figure 3).

\subsection{Effect of Intensity-Dependent Coupling Functional}

Comparing Figure 2(B), where we set the intensity coupling functional $f_{1}(n)=f_{2}(n)=1$ with cases considered in Figure 5(A) where we take $f_{1}(n)=f_{2}(n)=\sqrt{n}$, we note that the shape of the spectrum is changed on both sides of the central frequency. Also, there is no absorbtion or amplification at the central line. Furthermore, the range of the spectrum is larger and numbers of absorption and amplification component are increased, and the distance between them becomes larger than the case of $f_{1}(n)=f_{2}(n)=1$ (compare Figure 5(A) to Figure 2(B)). This due to, in this case the Rabi frequency is larger than that in the case $f_{1}(n)=f_{2}(n)=1$. But the situation is completely changed, when we take $f_{1}(n)=f_{2}(n)=1 / \sqrt{(n+1)}$. Since the Rabi frequency in this case is smaller than that in $f_{1}(n)=f_{2}(n)=1$, hence the range of 


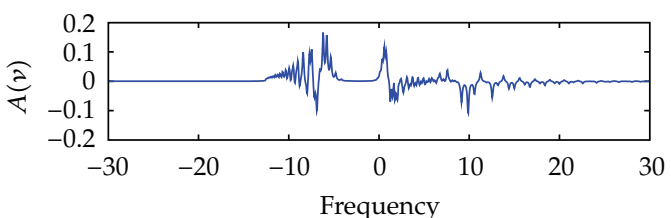

(a)

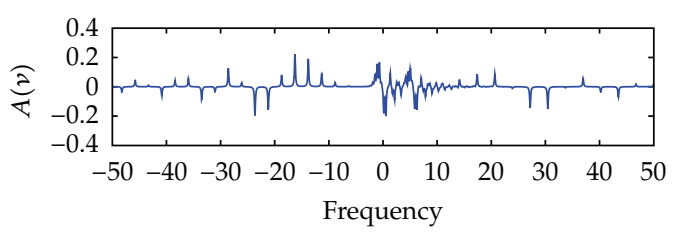

(b)

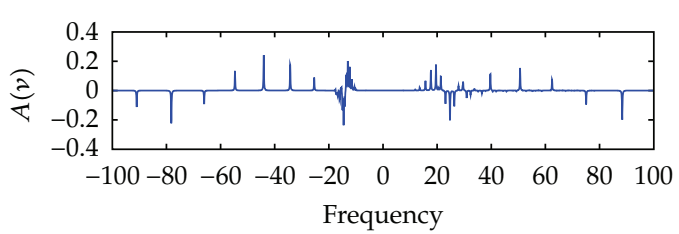

(c)

(A) Squeezed coherent field

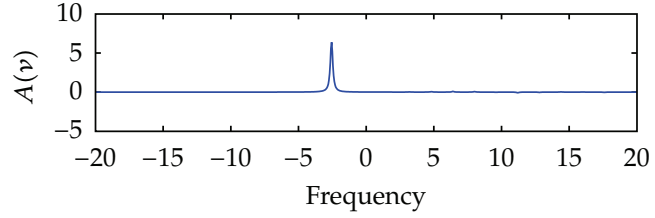

(a)

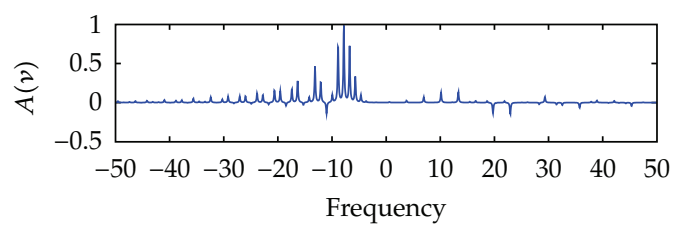

(b)

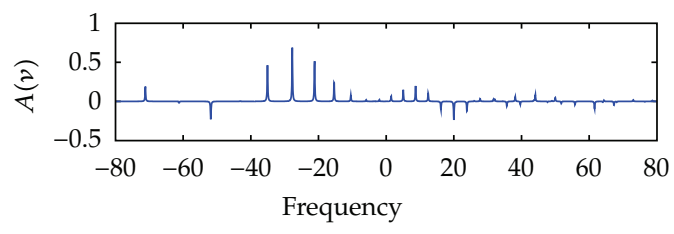

(c)

(B) Squeezed coherent field

Figure 4: The same as Figure 2(B) but with (1) $x=0.1$ and (2) $x=0.8$.

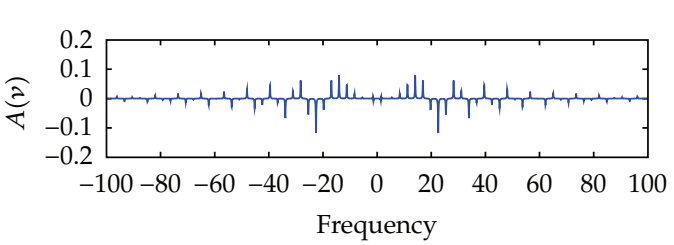

(a)

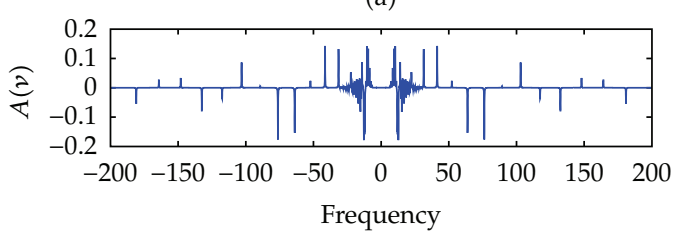

(b)

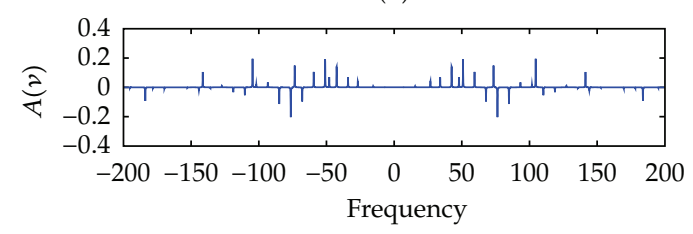

(c)

(A) Squeezed coherent field

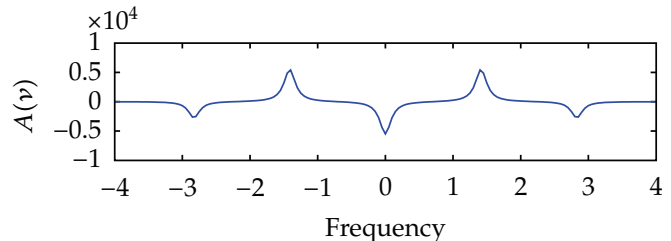

(a)

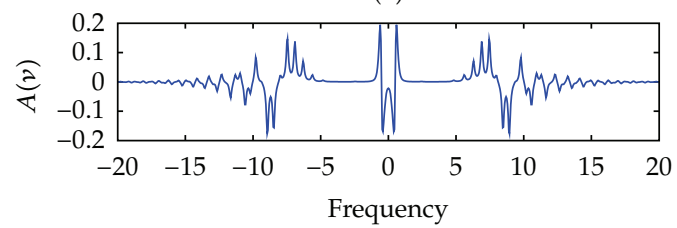

(b)

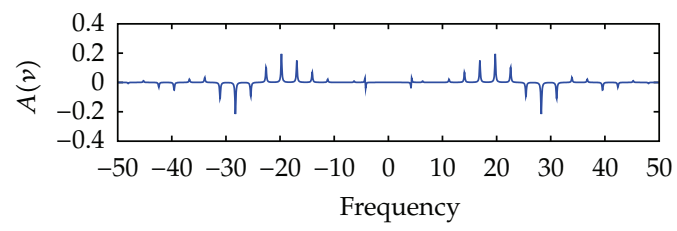

(c)

(B) Squeezed coherent field

Figure 5: The same as Figure 2(B) but (1) $f_{1}(n)=f_{2}(n)=\sqrt{n},(2) f_{1}(n)=f_{2}(n)=1 / \sqrt{n+1}$. 
the spectrum is smaller than that when we take $f_{1}(n)=f_{2}(n)=1$. Also, we observe that, the shape of the spectrum is completely changed where for $k=1$ we note a deep hole burning giving a strong amplification at the center of the spectrum surrounded by one absorption and one amplification peak, see Figure 5(B)-(a). This central hole burning disappearing for $k>1$, where the spectrum becomes rich and exhibits switch from amplification to absorption as $k$ increase (see Figures 5(B)-(b,c)). Finally, absorption spectrum can be controlled by choosing a right form of intensity-dependent coupling functional.

\section{Conclusion}

We have investigated the absorption spectrum for a multiphoton interaction with a $V$-type three-level atom, taking into account arbitrary forms of nonlinearities of both the field and the intensity-dependent atom-field coupling. The spectrum is calculated when the field is initially in a squeezed coherent state. We have explored the influence of various parameters of the system on the absorption spectrum. It is observed that

(i) as the photon multiplicities number increase, the number of allowed transition between the dressed states increases and hence number of absorption and amplification peaks appearing in the spectrum increases as $k$ increases;

(ii) the position of absorption and amplification peaks is associated with not only the photon number $(\bar{n})$ and the photon multiplicity number $k$ but also the intensitydependent atom-field coupling $\lambda_{i} f_{i}(n)$;

(iii) the heights of the spectrum components becomes shorter and the distances between them is larger as the mean photon number increases;

(iv) the symmetry shown in the standard three-level atom model for the spectra is no longer present once Kerr effect or detuning is considered;

(v) the effect of detuning on the absorption spectrum is twofold. The first effect is the shift of the spectrum to the right hand side. The second effect is the dependence of the amplitudes and heights of the peaks on $\Delta_{i}$;

(vi) the Kerr medium has an effect opposite to the effect of the detuning, where the earlier has shorter elements. Also, the heights and widths of the peaks not only depend on the photon multiplicity but also depend on the value of $x$. Consequently, changes in the detuning and the Kerr medium parameters can show in the spectra, and hence the heights of the peaks; their shifts and widths are altered when compared with the case of resonance;

(vii) the strong field effects can be produced by choosing the right parameters for these nonlinearities.

\section{References}

[1] C. Hooijer, G. X. Li, K. Allaart, and D. Lenstra, "Spontaneous emission in a V-type three-level atom driven by a classical field," Physics Letters, Section A, vol. 263, no. 4-6, pp. 250-256, 1999.

[2] Z. Ficek and P. D. Drummond, "Three-level atom in a broadband squeezed vacuum field-I. General theory," Physical Review A, vol. 43, no. 11, pp. 6247-6257, 1991.

[3] S. Smart and S. Swain, "Three-level atom in a squeezed vacuum-II. Resonance fluorescence," Journal of Modern Optics, vol. 41, no. 6, pp. 1055-1077, 1994. 
[4] B. J. Dalton, M. R. Ferguson, and Z. Ficek, "Resonance fluorescence spectra of three-level atoms in a squeezed vacuum," Physical Review A, vol. 54, no. 3, pp. 2379-2390, 1996.

[5] B. J. Dalton, M. Bosticky, and Z. Ficek, "Probe absorption spectra for driven atomic systems in a narrow bandwidth squeezed vacuum," Physical Review A, vol. 53, no. 6, pp. 4439-4467, 1996.

[6] F. Carreño, M. A. Antón, and O. G. Calderón, "Quantum interference effects in resonance fluorescence and absorption spectra of a V-type three-level atom damped by a broadband squeezed vacuum," Optics Communications, vol. 221, no. 4-6, pp. 365-385, 2003.

[7] D. F. Walls and G. J. Milburn, Quantum Optics, Springer, Berlin, Germany, 2nd edition, 2008.

[8] C. M. Caves and B. L. Schumaker, "New formalism for two-photon quantum optics-I. Quadrature phases and squeezed states," Physical Review A, vol. 31, no. 5, pp. 3068-3092, 1985.

[9] V. V. Dodonov, "Nonclassical states in quantum optics: a squeezed review of the first 75 years," Journal of Optics B. Quantum and Semiclassical Optics, vol. 4, no. 1, pp. R1-R33, 2002.

[10] J. Peřina, Quantum Statistics of Linear and Nonlinear Optical Phenomena, D. Reidel, Dordrecht, The Netherlands, 1984. 


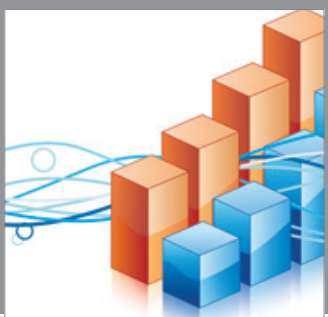

Advances in

Operations Research

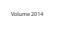

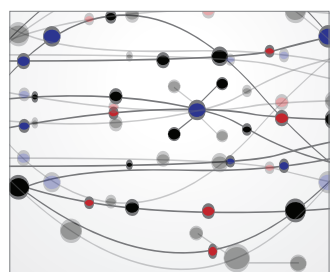

\section{The Scientific} World Journal
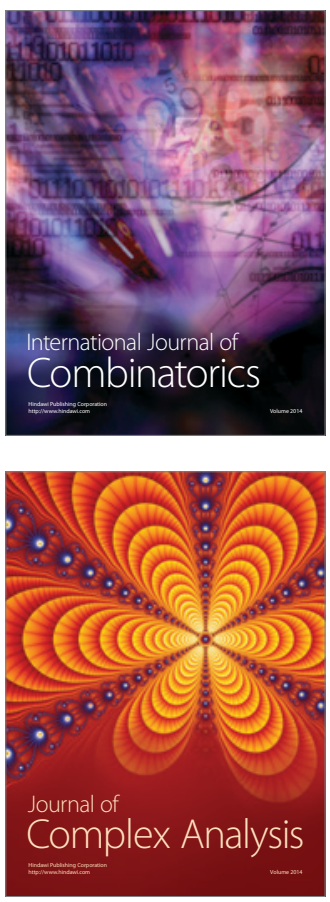

International Journal of

Mathematics and

Mathematical

Sciences
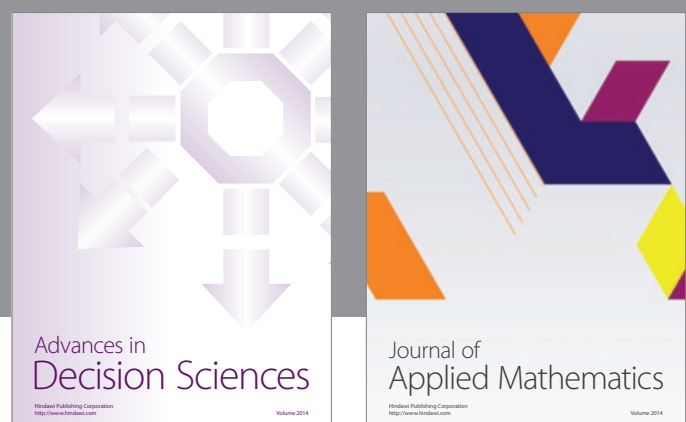

Journal of

Applied Mathematics
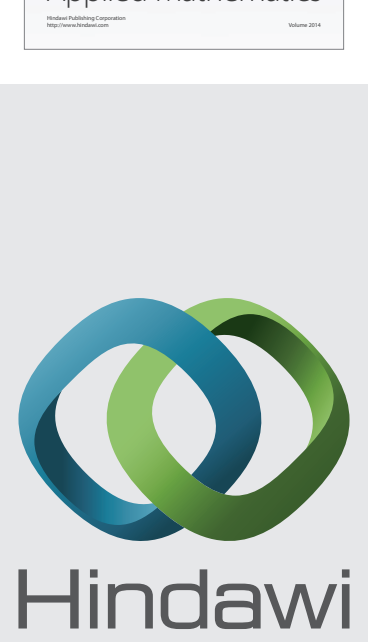

Submit your manuscripts at http://www.hindawi.com
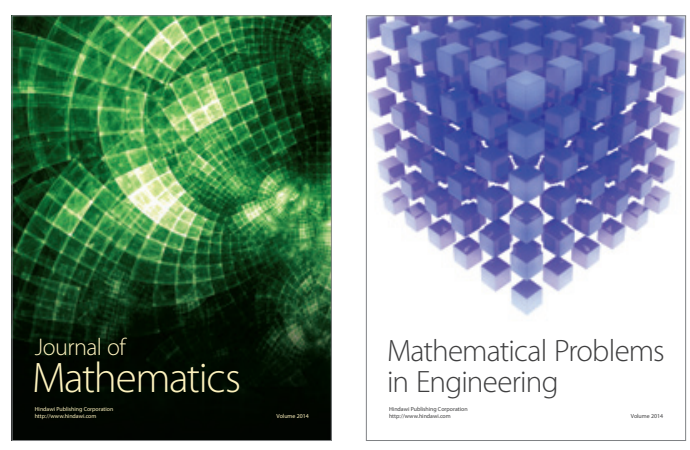

Mathematical Problems in Engineering
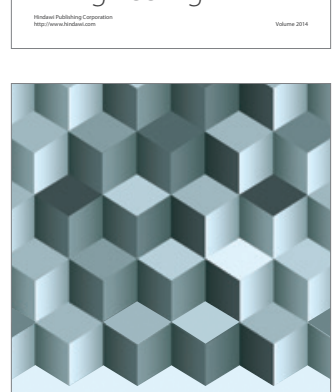

Journal of

Function Spaces
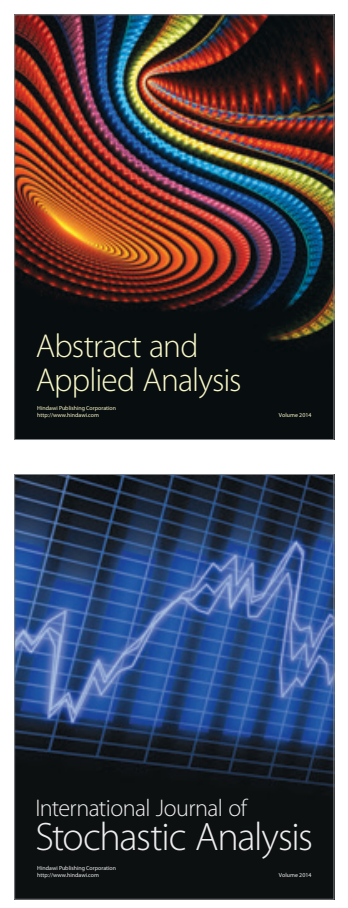

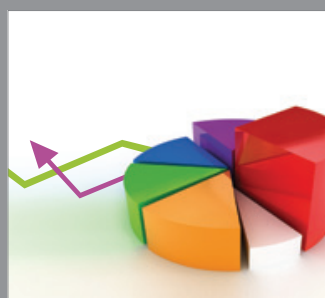

ournal of

Probability and Statistics

Promensencen
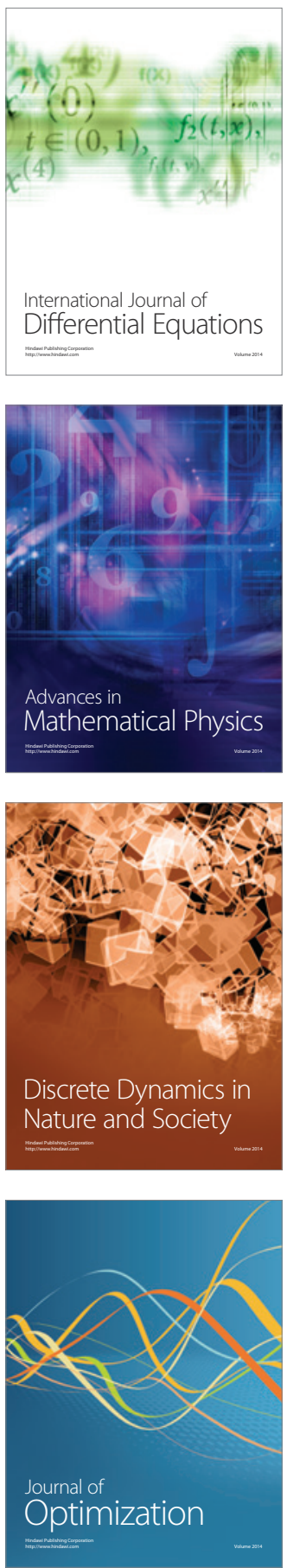